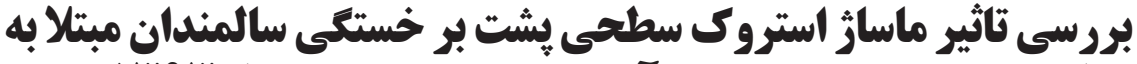

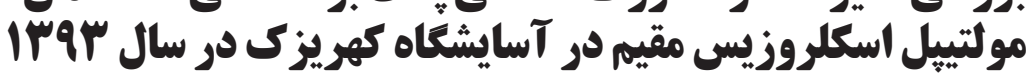

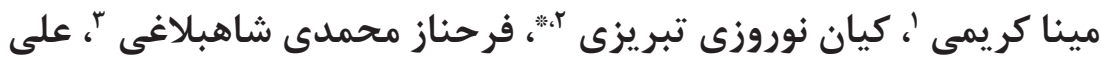

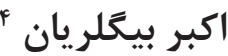

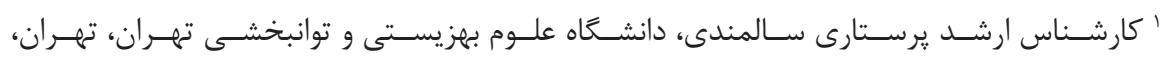

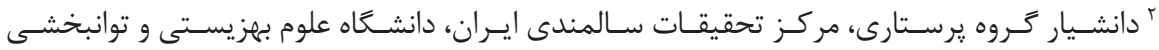

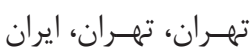

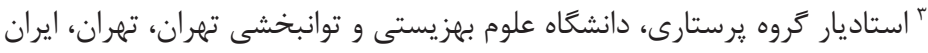

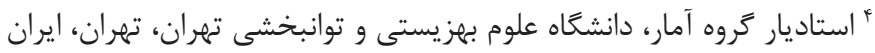

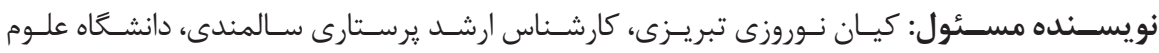

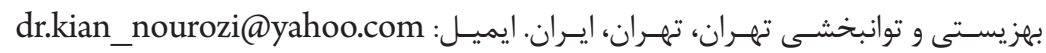

DOI: $10.21859 /$ ijrn-02047

קفكيده

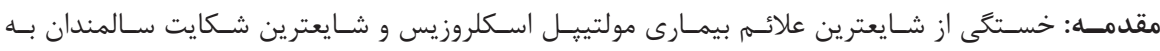

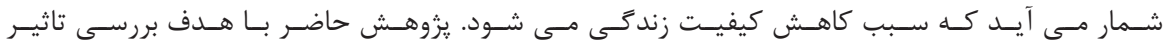

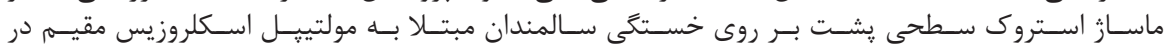

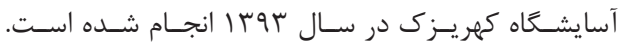

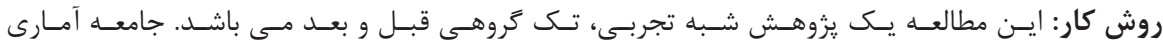

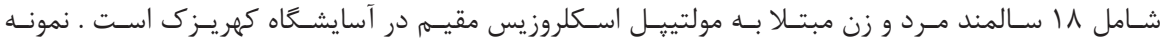

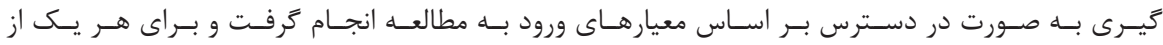

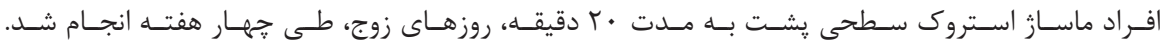

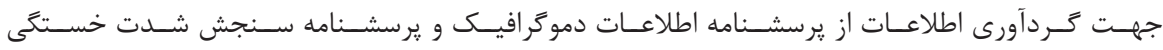

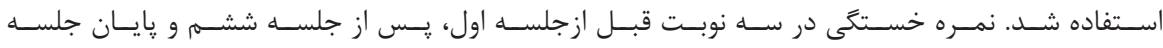

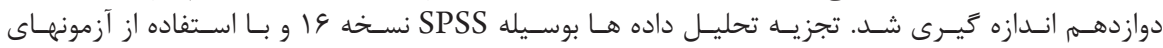

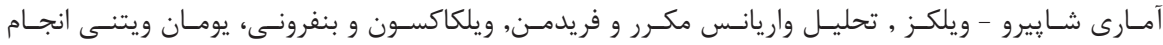

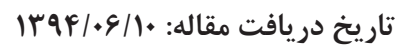
تاريخ يذيرش مقاله: تاريخ درت مقاله:

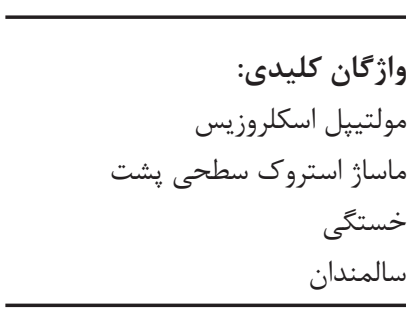

تمامى حقوق نشر براى انجمن علمى يرستارى

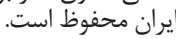

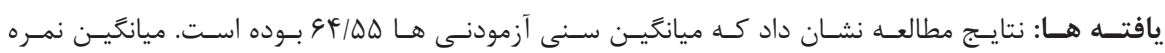

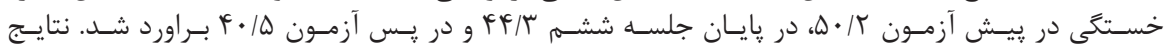

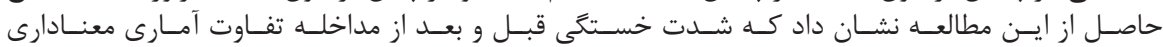

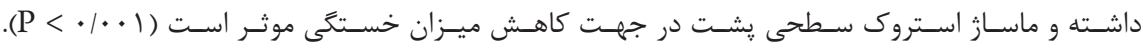

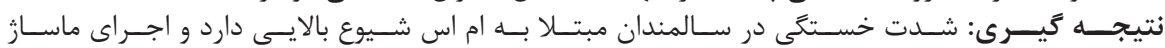

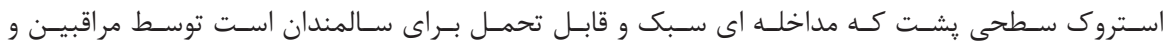

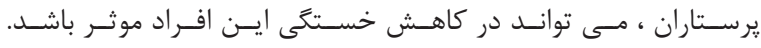

افزايـش يافتـه و ضـرورت توجـهـ بـه مشـكلات آنـان بيـش از بيـش

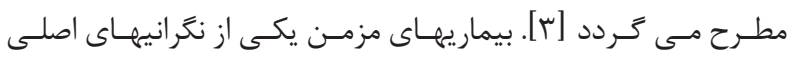

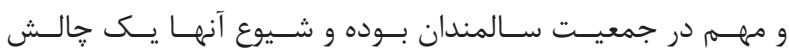

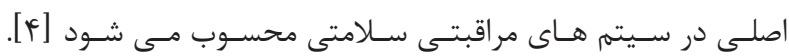

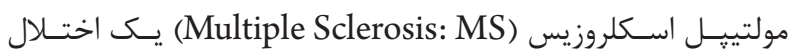

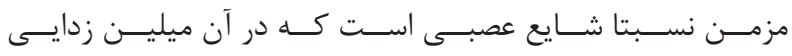

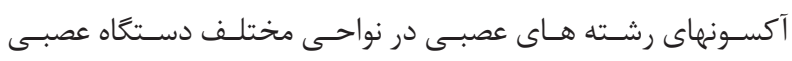

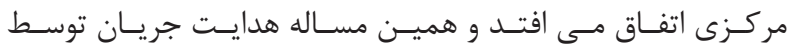

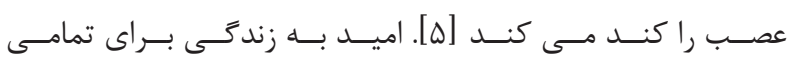

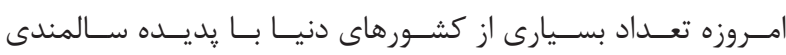

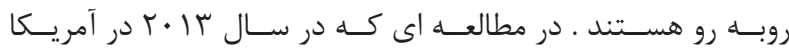

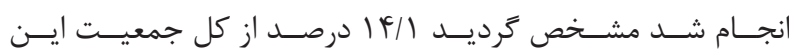

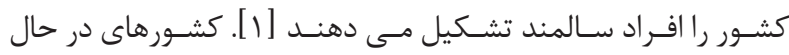

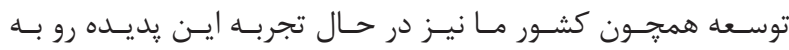

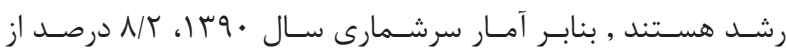

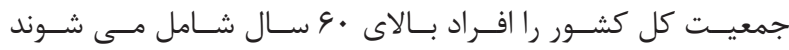

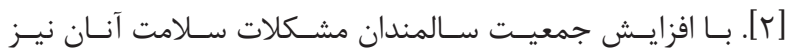


بخشى از برنامسه مر اقبتى -بهبودى (Caring-Healing) شـده اسـت

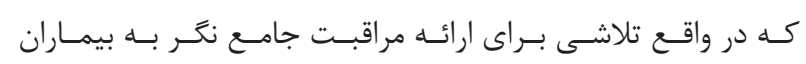

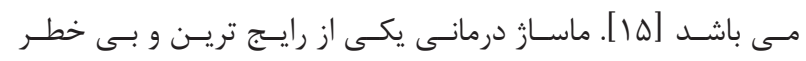

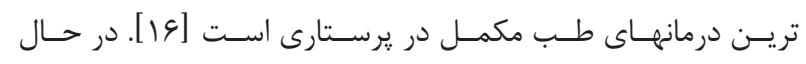

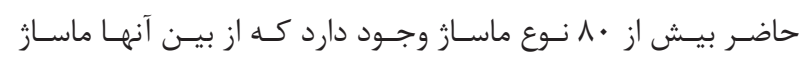

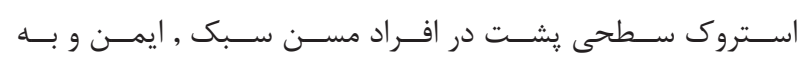

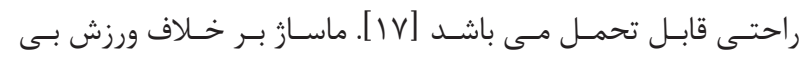

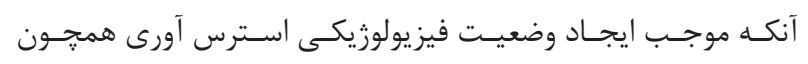

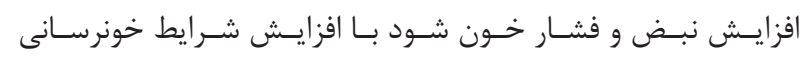

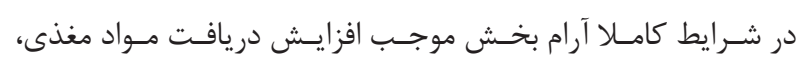

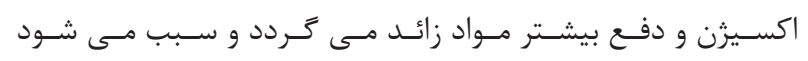

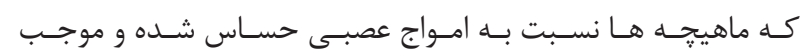

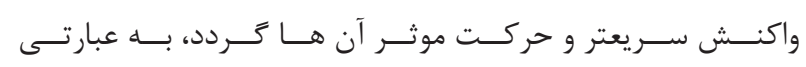

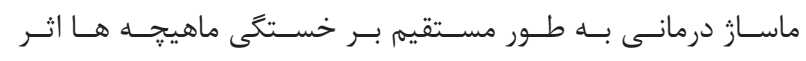

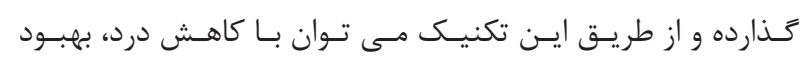

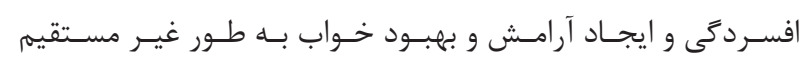

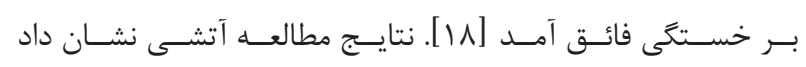

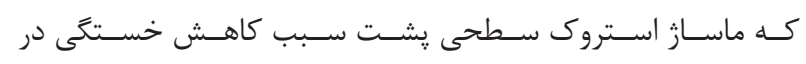

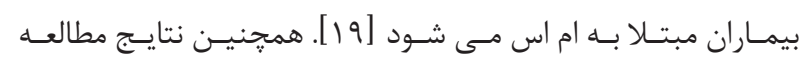

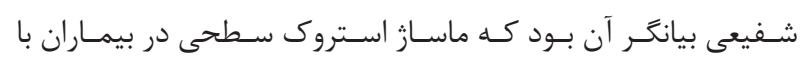

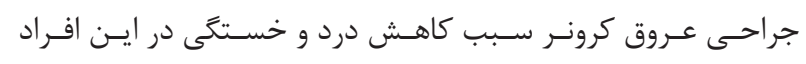

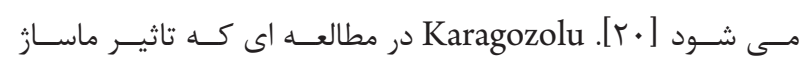

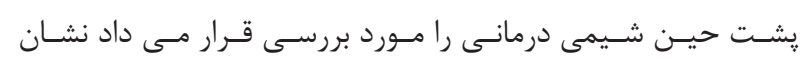

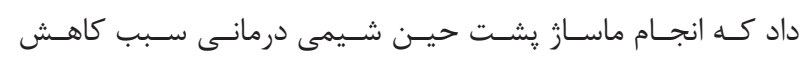

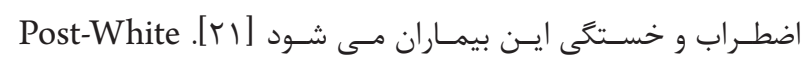

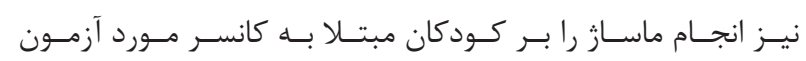

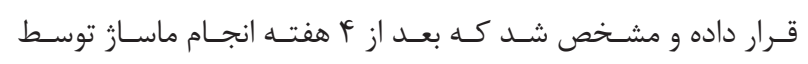

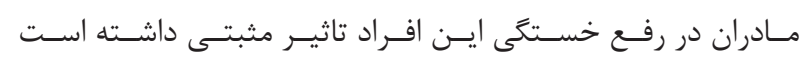

[rr]

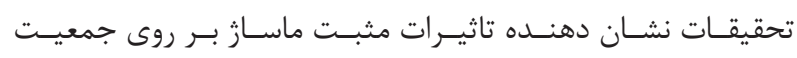

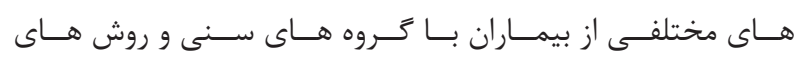

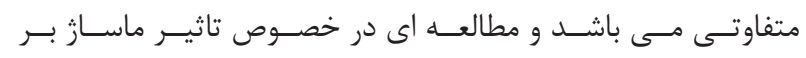

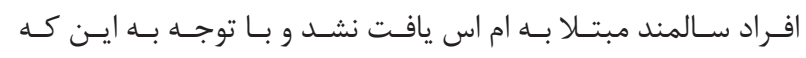

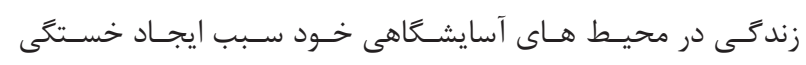

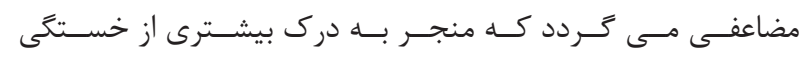

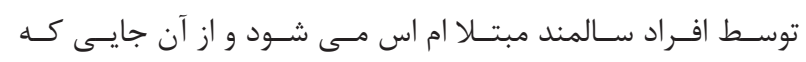

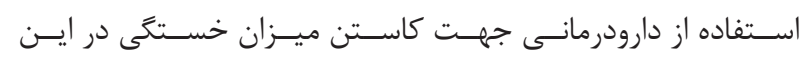

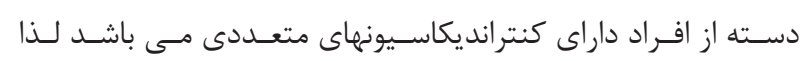

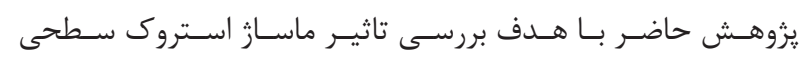

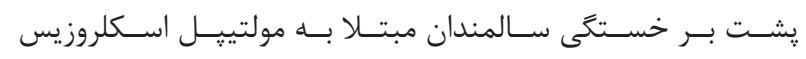

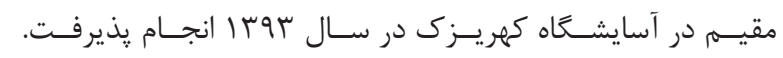

بيمـاران بـا اشـكال متفــاوت ام اس نسـبتا طبيعسى بـوده و افـراد

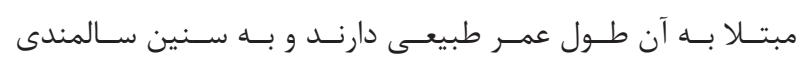

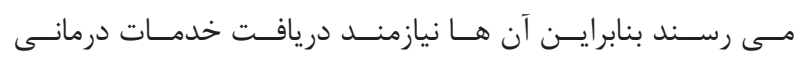

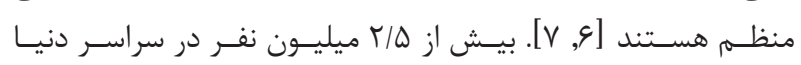

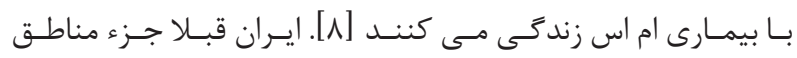

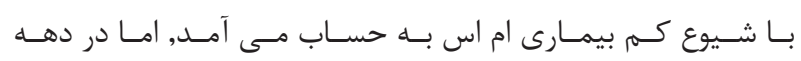

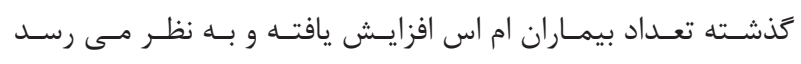

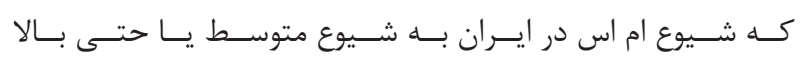

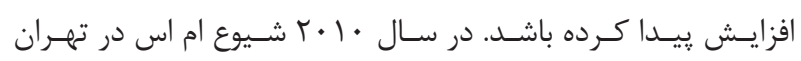

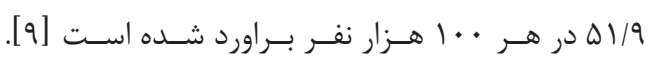

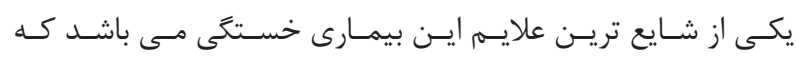

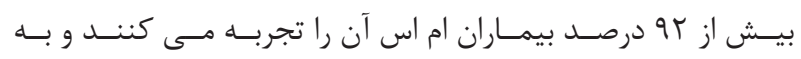

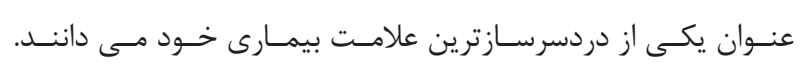

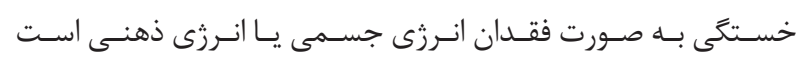

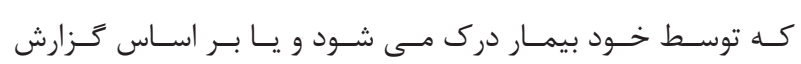

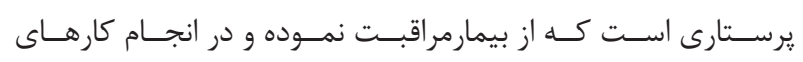

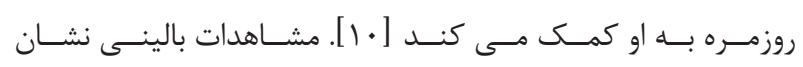

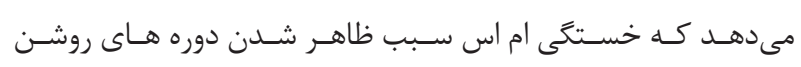

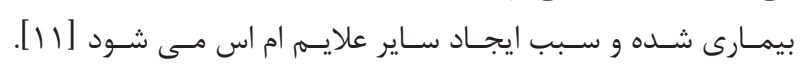

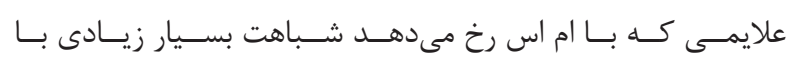

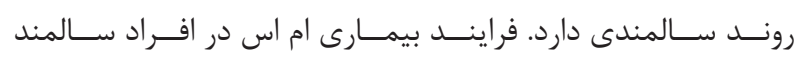

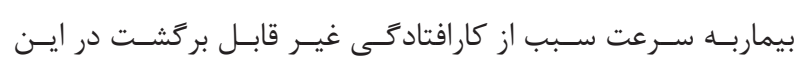

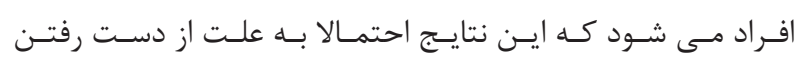

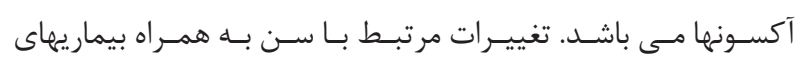

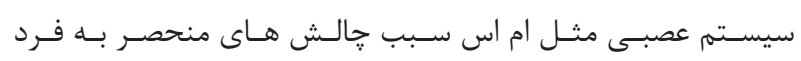

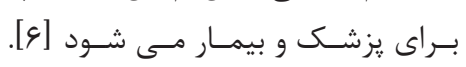

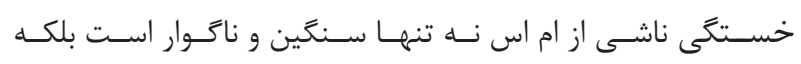

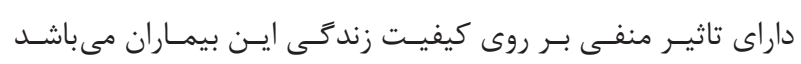

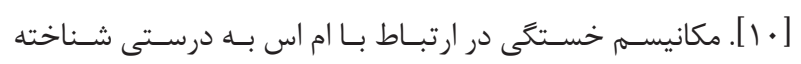

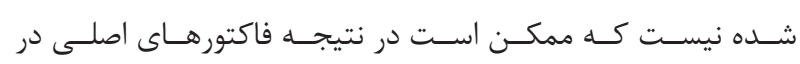

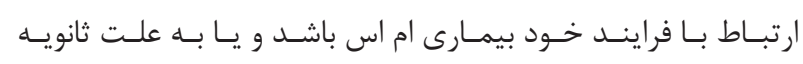

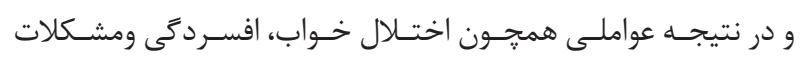

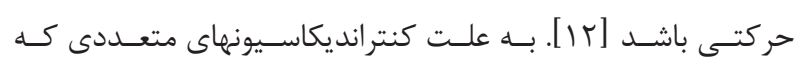

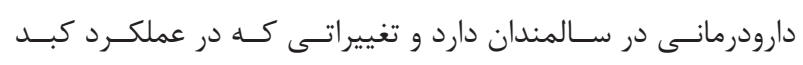

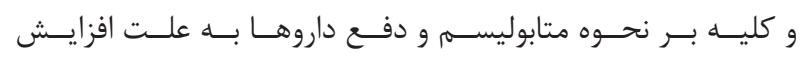

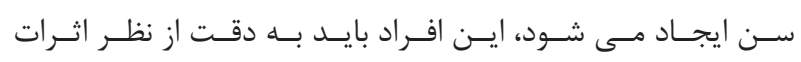

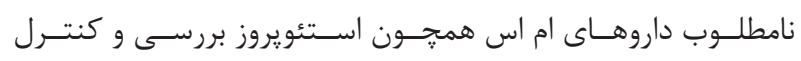

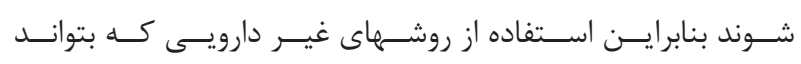

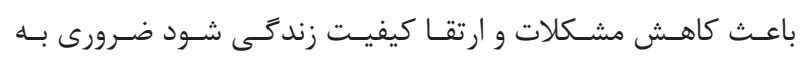

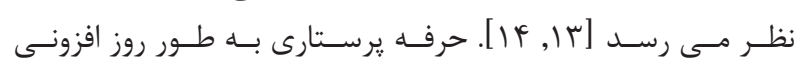

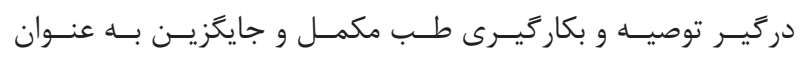




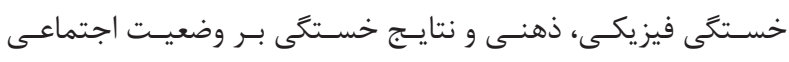

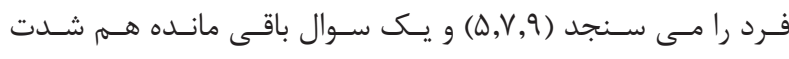

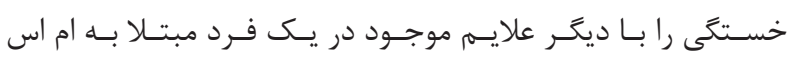

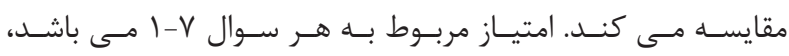

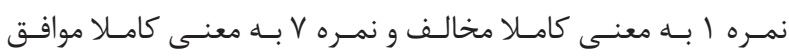

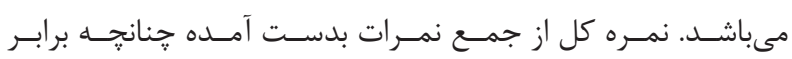

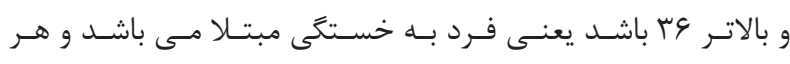

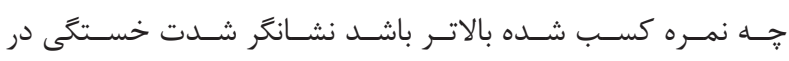

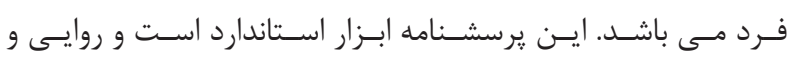

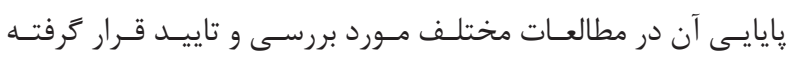

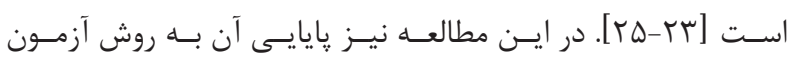
مجـد

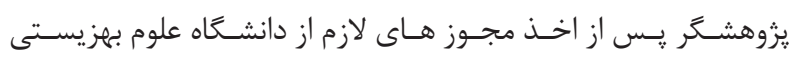

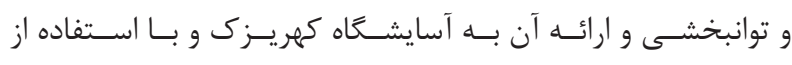

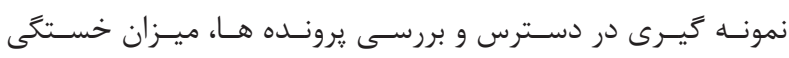

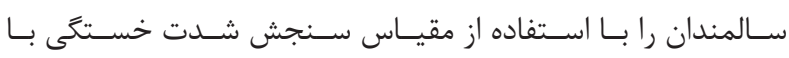

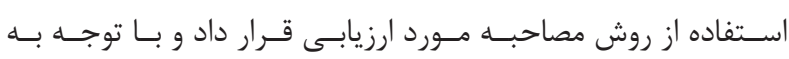

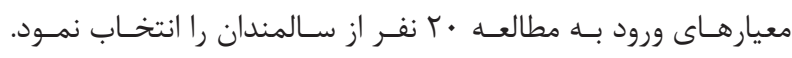

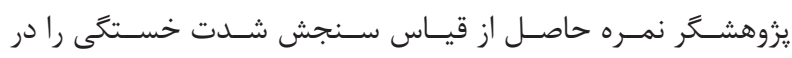

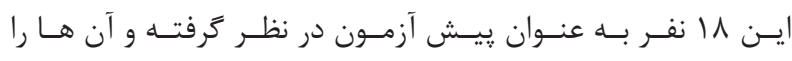

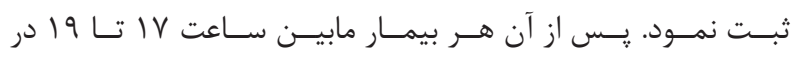

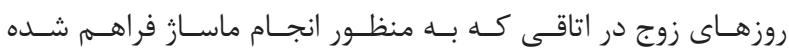

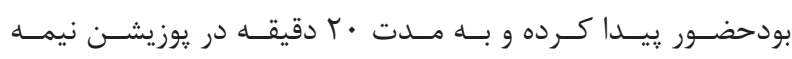

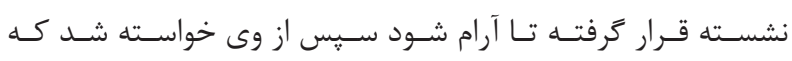

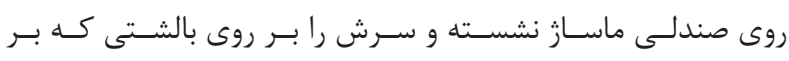

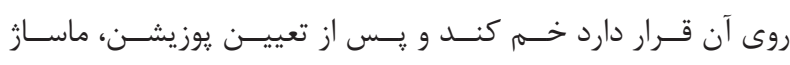

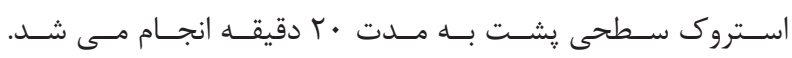

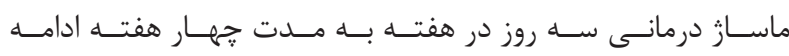

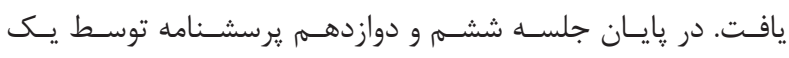

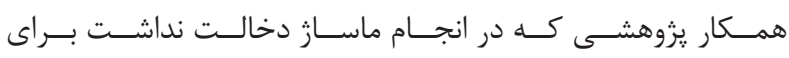

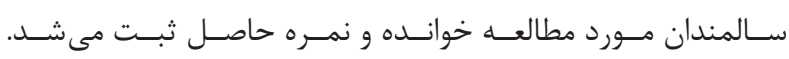

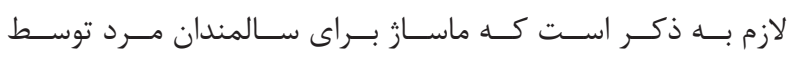

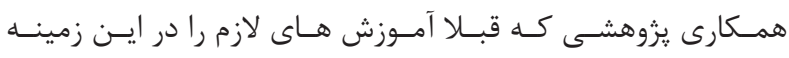

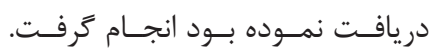

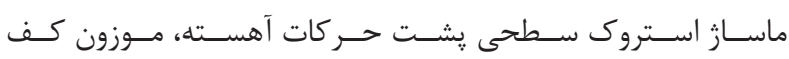

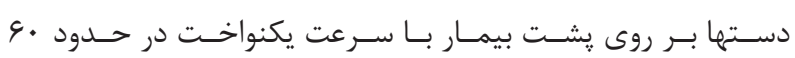

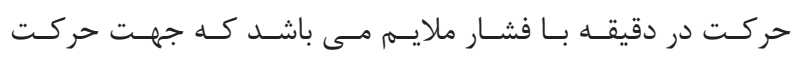

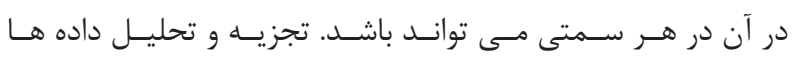

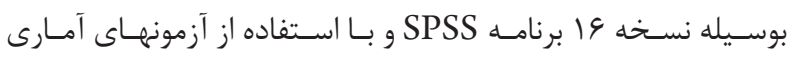

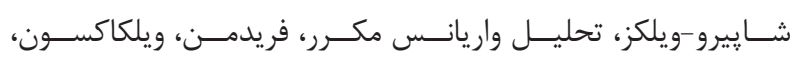

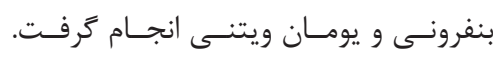

\section{روش كار}

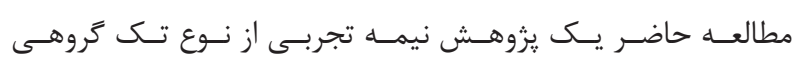

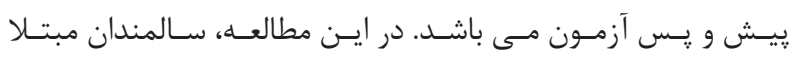

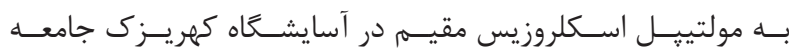

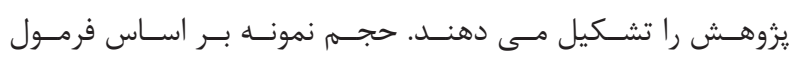

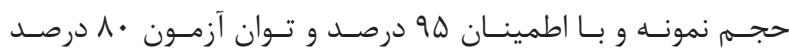

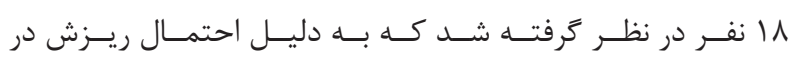

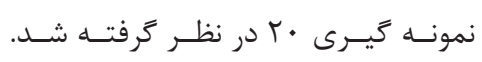

$$
n=\frac{\left(z_{1}-\frac{\alpha}{2}+z_{1}-\beta\right)^{2} \cdot s^{2} p}{2}
$$

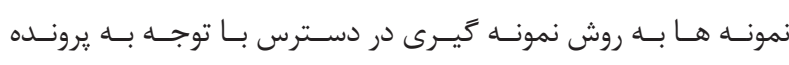

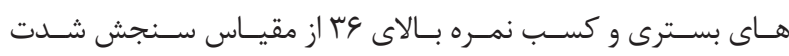

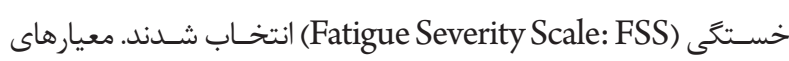

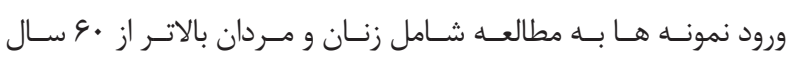

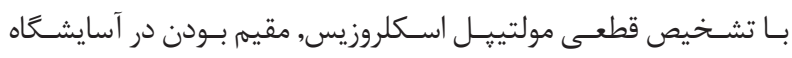

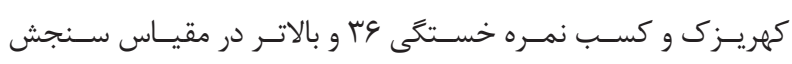

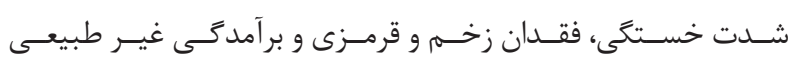

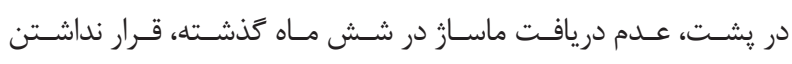

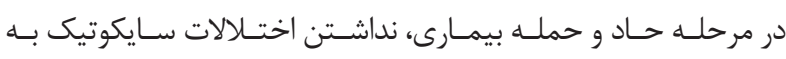

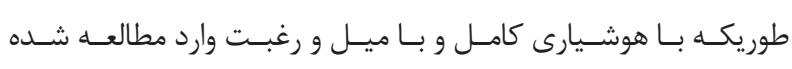

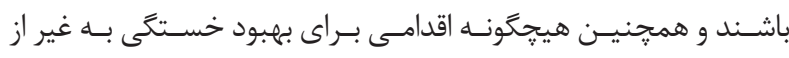

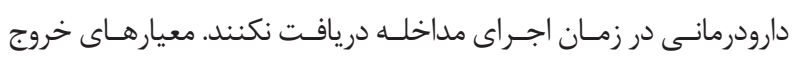

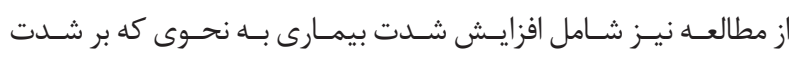

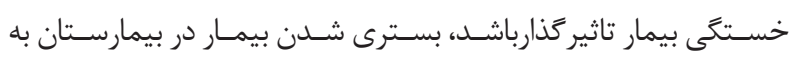

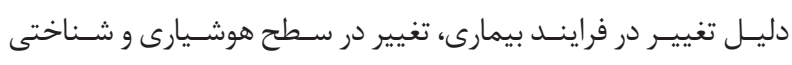

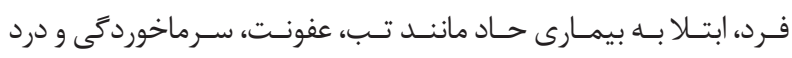

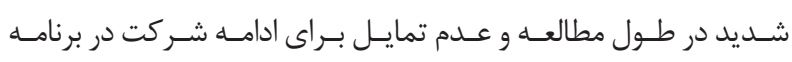

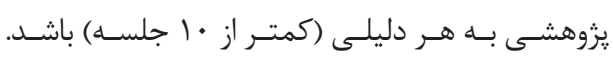

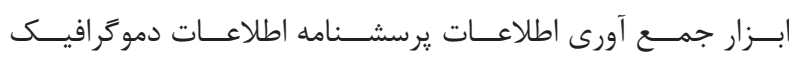

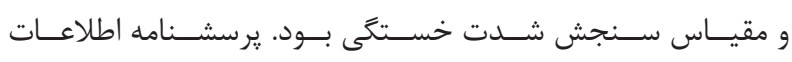

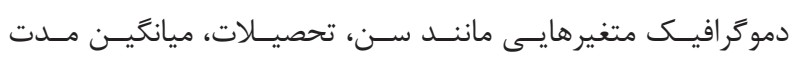

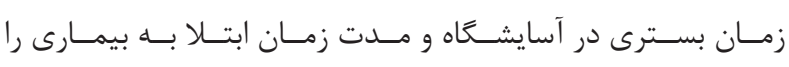

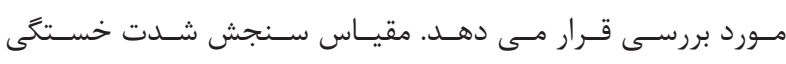

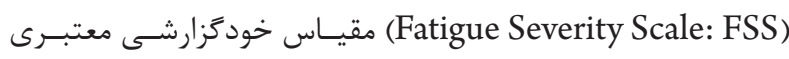

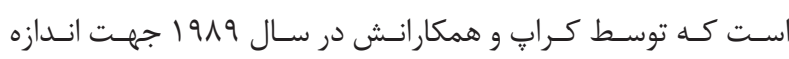

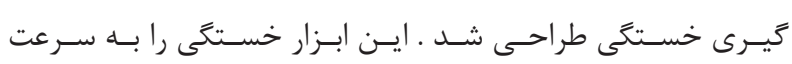

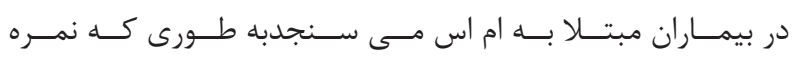

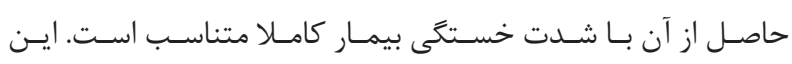

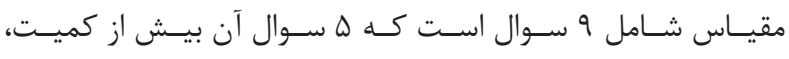

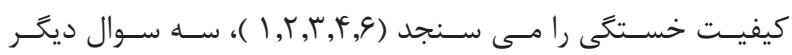




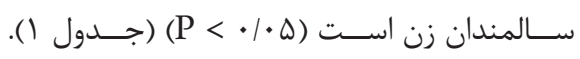

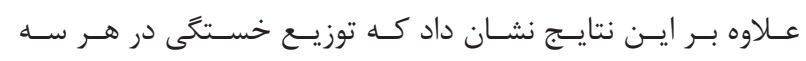

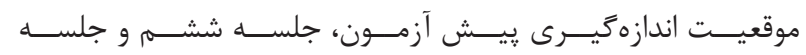

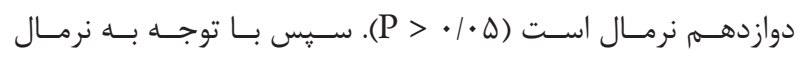

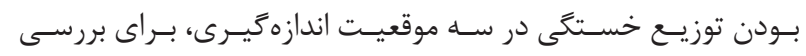

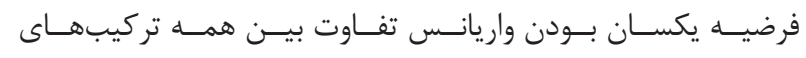

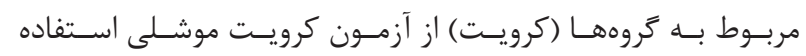

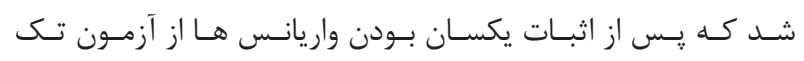

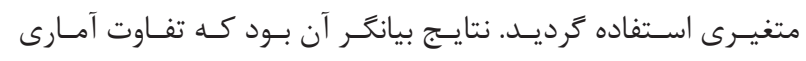

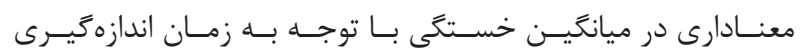

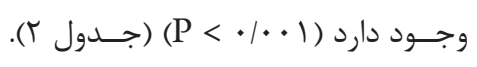

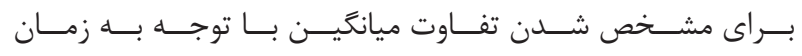

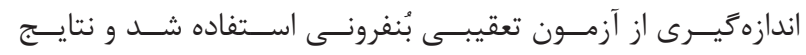

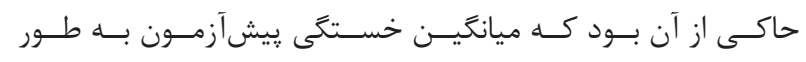

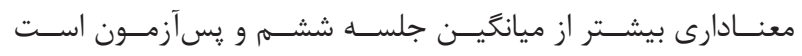

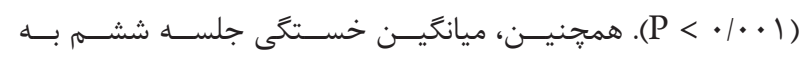

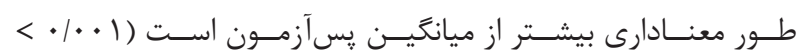

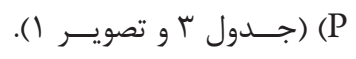

\section{يافتهها}

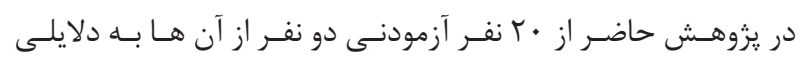

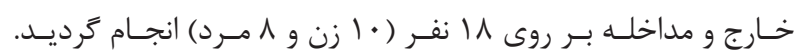

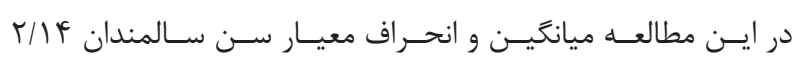

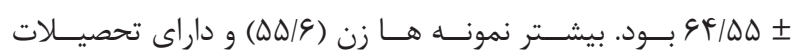

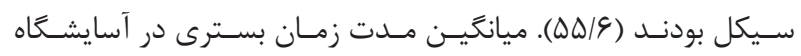

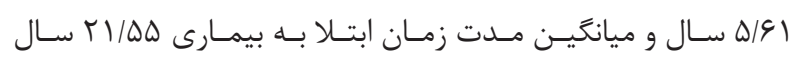

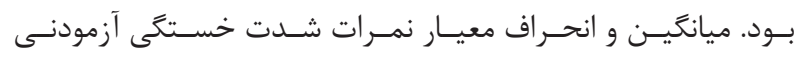

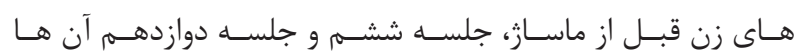

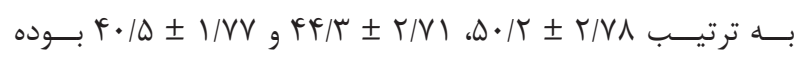

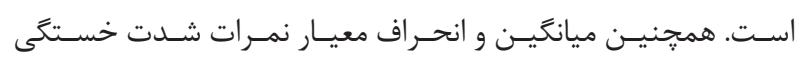

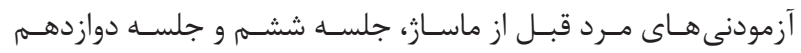
آن هـا بـه ترتيسب

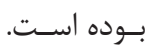

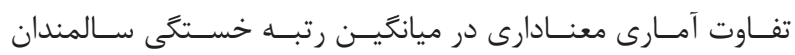

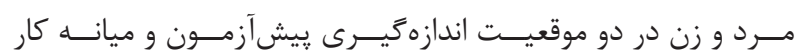

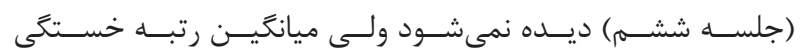

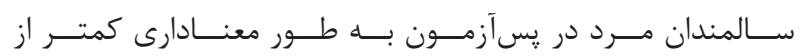

\begin{tabular}{|c|c|c|c|c|c|c|c|c|}
\hline \multicolumn{9}{|c|}{ جدام مداخله ا:مقايسه ميانكَين خستخى سالمند مرد و زن مبتلا به مولتيبل اسكلروزيس مقيم آسايشكاه كهريزك شركت كننده در يروهش قبل و بعد از } \\
\hline ن و ويتنى يو & 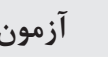 & & زن & & & مرد & & موقعيت اندازهكيرى \\
\hline مقدار احتمال & آماره & انحرافمعيار & ميانگَين & تعداد & انحرافمعيار & ميانگَين & تعداد & \\
\hline.$/ 9 Y \Lambda$ & -.1 .91 & T/VA & $\Delta \cdot / T$ & 1. & T/19 & $\Delta \cdot / 1 T$ & $\wedge$ & ييش آزمون \\
\hline - IATT & $-\cdot / T T \Delta$ & $T / V I$ & 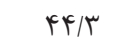 & 1. & 1/Ar & $F F / T D$ & $\wedge$ & جلسه ششم \\
\hline$\cdot \mid \cdot+4$ & $-r / \cdot F$ & $1 / V V$ & $r \cdot 1 \Delta$ & 1. & $1 / \Gamma \Lambda$ & rN/VQ & $\wedge$ & يس آزمون \\
\hline
\end{tabular}

جدول rا: ميانكَين و انحراف معيار خستخى سالمندان مبتلا به مولتيبل اسكلروزيس مقيم آسايشكاه كهريزك شركت كننده در ثروهش قبل و بعد از انجام مداخله موقعيت اندازهيرى تعداد كمترين بيشترين ميانكين انحرافمعيار آزمون تك متغيرى تحليل واريانس مكرر آماره مقدار احتمال اندازه اثر

\begin{tabular}{|c|c|c|c|c|c|c|c|c|}
\hline & & & $r / F \Delta$ & $\Delta \cdot / 19$ & $\Delta \Delta$ & is & 11 & يِيش آزمون \\
\hline \multirow[t]{2}{*}{.$/ 941$} & $>\cdot \mid \cdot .1$ & $r \Delta \Lambda / q 1$ & $r / r q$ & $F F / T V$ & $p q$ & rq & 11 & جلسه ششم \\
\hline & & & $1 / V V$ & rq/vr & Fr & rv & 11 & يس آزمون \\
\hline
\end{tabular}




\begin{tabular}{|c|c|c|c|}
\hline \multicolumn{4}{|c|}{ 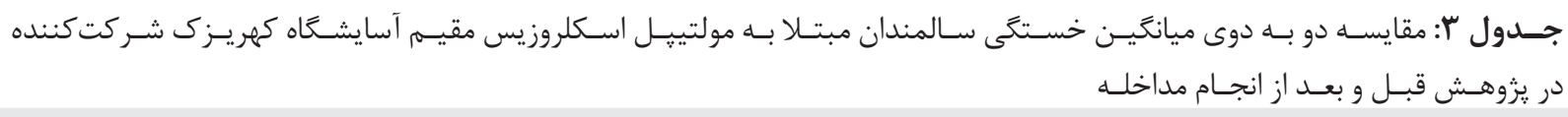 } \\
\hline مقدار احتمال & اختلاف ميانغين & موقعيت مورد مقايسه & موقعيت اندازهكيرى \\
\hline$<\cdot|\cdot|$ & $\Delta / \wedge \Lambda$ & جلسه ششم & ييش آزمون \\
\hline$<\cdot|\cdot \cdot|$ & $1 . / 44$ & سِ & \\
\hline$<\cdot 1 \cdot \cdot 1$ & $r / \Delta \Delta$ & سֶ آزمون & جلسه ششم \\
\hline
\end{tabular}

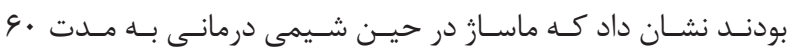

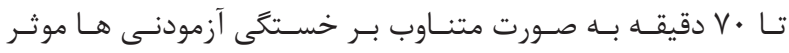

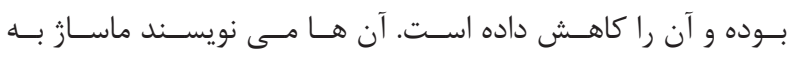

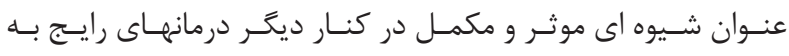

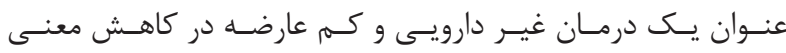

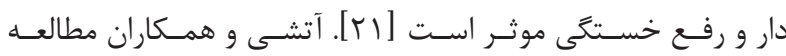

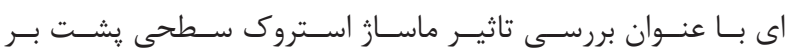

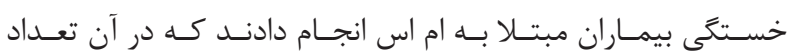

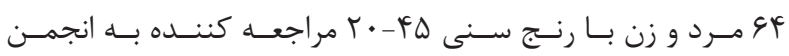

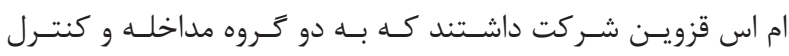

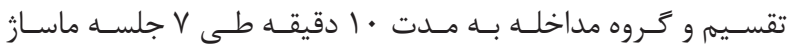

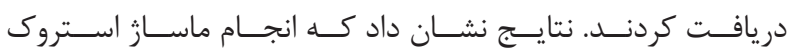

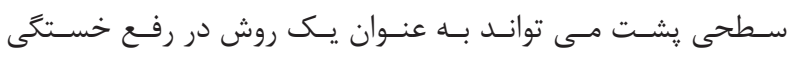

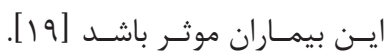

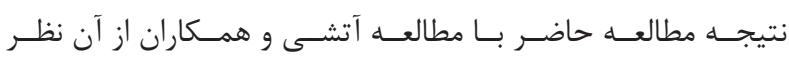

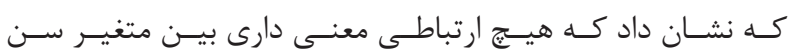

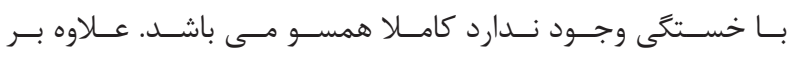

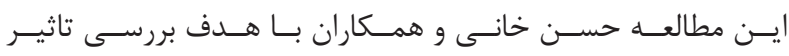

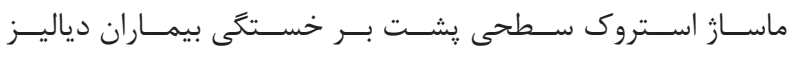

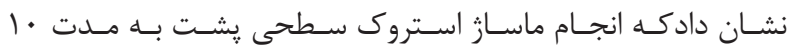

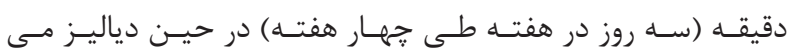

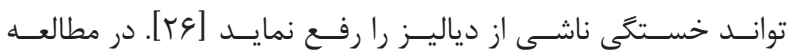

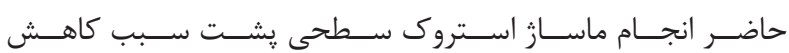

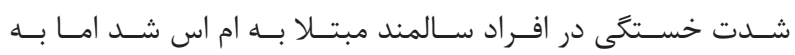

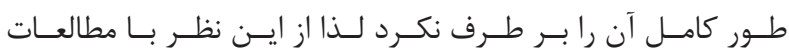

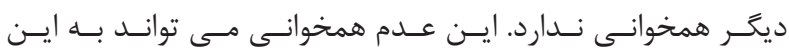

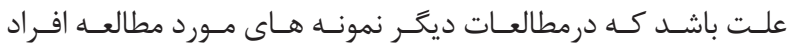

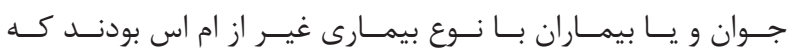

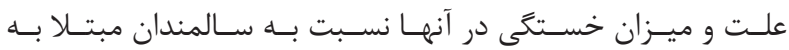

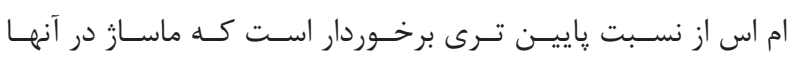

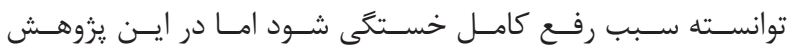

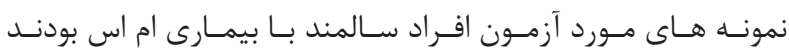

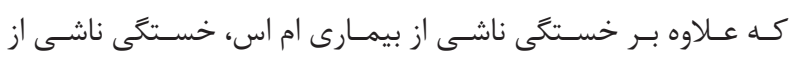

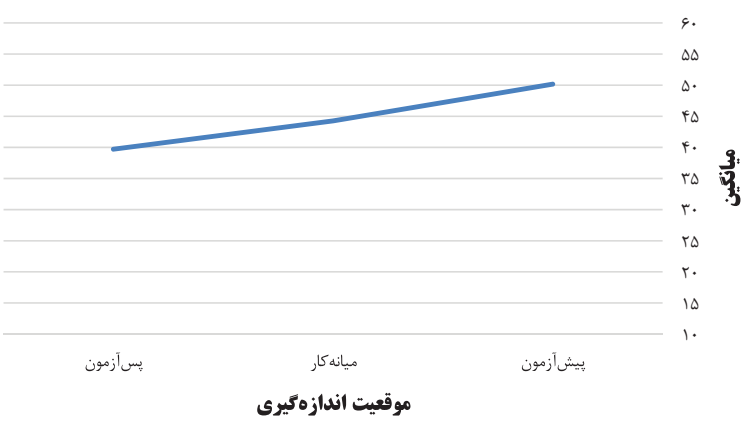

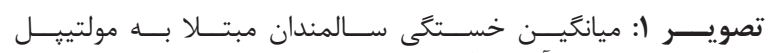

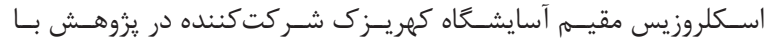

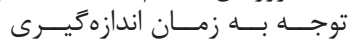

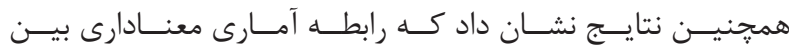

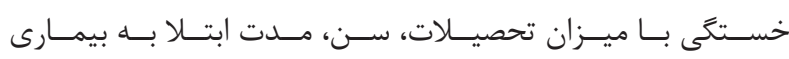

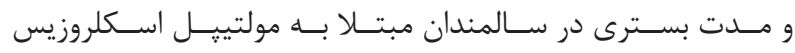

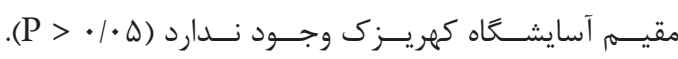

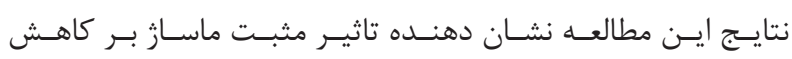

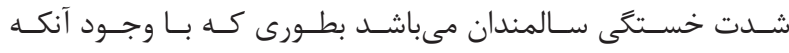

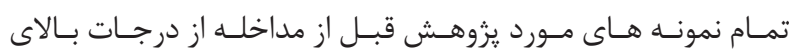

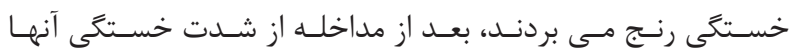

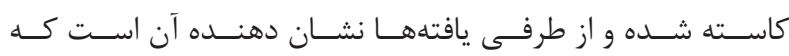

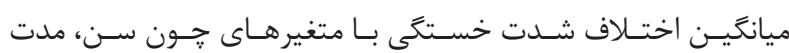

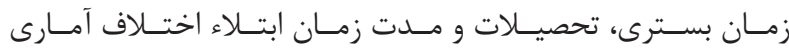

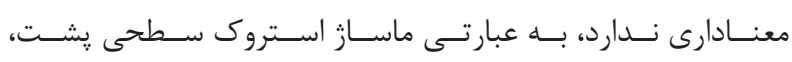

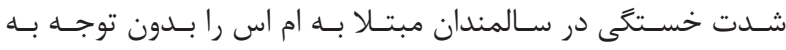

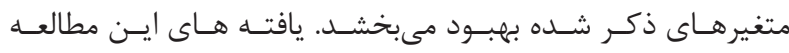

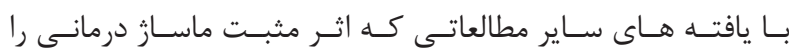

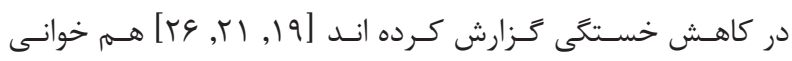
دارد. Karagozolu و همـكاران كـه مطالعـه اى را بـا هـــف بررسـى تاثير

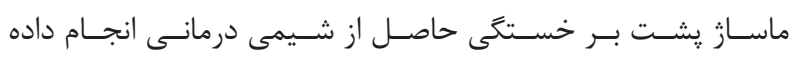


بررسـى راهكارهـاى جهـت كاسـتن از ميـزان خسـتخى در ايـن گروه

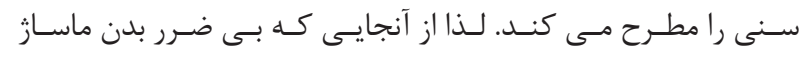

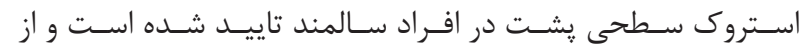

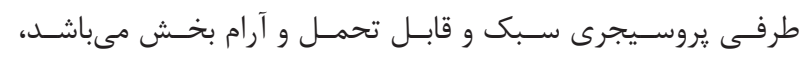

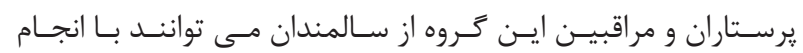

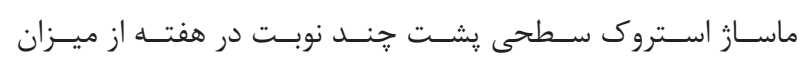

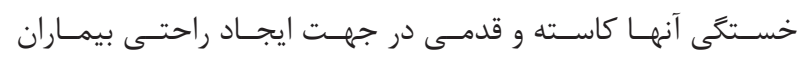
سـالمند مبتـلا بـهـ ام اس بردارنـد.

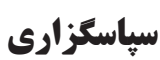

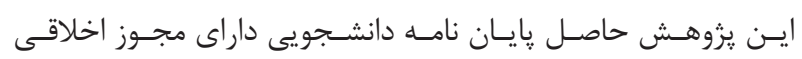

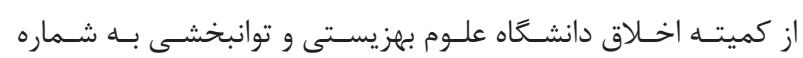
IRCT2015092524182N1 و و كـد USWR.REC.1393.235

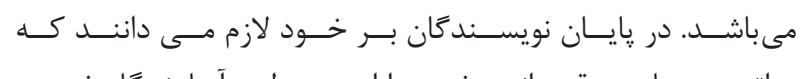

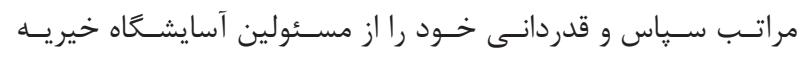

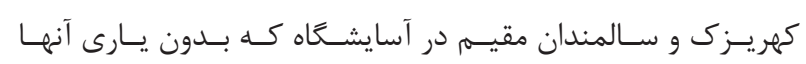

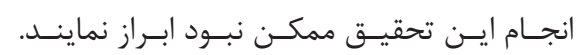

تضاد منافع

هيجگُونه تضاد منافعى براى اين مقاله وجود ندارد.

\section{REFERENCES}

1. Administration on Aging (AoA), Aging Statistics 2016 [updated 2016; cited 2016]. Available from: http://www.aoa.acl.gov/Aging_Statistics/ index.aspx.

2. Statistical Center of Iran 2016 [updated 2016; cited 2016]. Available from: www.amar.org.ir.

3. Coleman S, McQuade J, Rose J, Inderjeeth C, Carroll G, Briffa NK. Self-management for osteoarthritis of the knee: does mode of delivery influence outcome? BMC Musculoskelet Disord. 2010;11:56. DOI: 10.1186/1471-2474-11-56 PMID: 20334688

4. Puia D, McDonald DD. Older black adult osteoarthritis pain communication. Pain Manag Nurs. 2014;15(1):229-35. DOI: 10.1016/j. pmn.2012.09.001 PMID: 23141080

5. Brunner LS, Suddarth DS, Smeltzer SCOC, Bare BG. Brunner \& Suddarth's Textbook of Medical-surgical Nursing: Lippincott Williams \& Wilkins; 2004.

6. Stern M. Aging with multiple sclerosis. Phys Med Rehabil Clin N Am. 2005;16(1):219-34. DOI: 10.1016/j.pmr.2004.06.010 PMID: 15561552

7. MacAllister WS, Krupp LB. Multiple sclerosis-related fatigue. Phys Med Rehabil Clin N Am. 2005;16(2):483-502. DOI: 10.1016/j. pmr.2005.01.014 PMID: 15893683

8. Cameron MH, Poel AJ, Haselkorn JK, Linke A, Bourdette D. Falls requiring medical attention among veterans with multiple sclerosis: a cohort study. J Rehabil Res Dev. 2011;48(1):13-20. PMID: 21328159

9. Sahraian MA, Khorramnia S, Ebrahim MM, Moinfar Z, Lotfi J, Pakdaman H. Multiple sclerosis in Iran: a demographic study of 8,000 patients and changes over time. Eur Neurol. 2010;64(6):331-6. DOI: 10.1159/000321649 PMID: 21071949

10. Amato MP, Portaccio E. Management options in multiple sclerosis-associated fatigue. Expert Opin Pharmacother. 2012;13(2):207-16. DOI: 10.1517/14656566.2012.647767 PMID: 22220738

11. Krupp LB, Serafin DJ, Christodoulou C. Multiple sclerosis-associated fatigue. Expert Rev Neurother. 2010;10(9):1437-47. DOI: 10.1586/ ern.10.99 PMID: 20819014

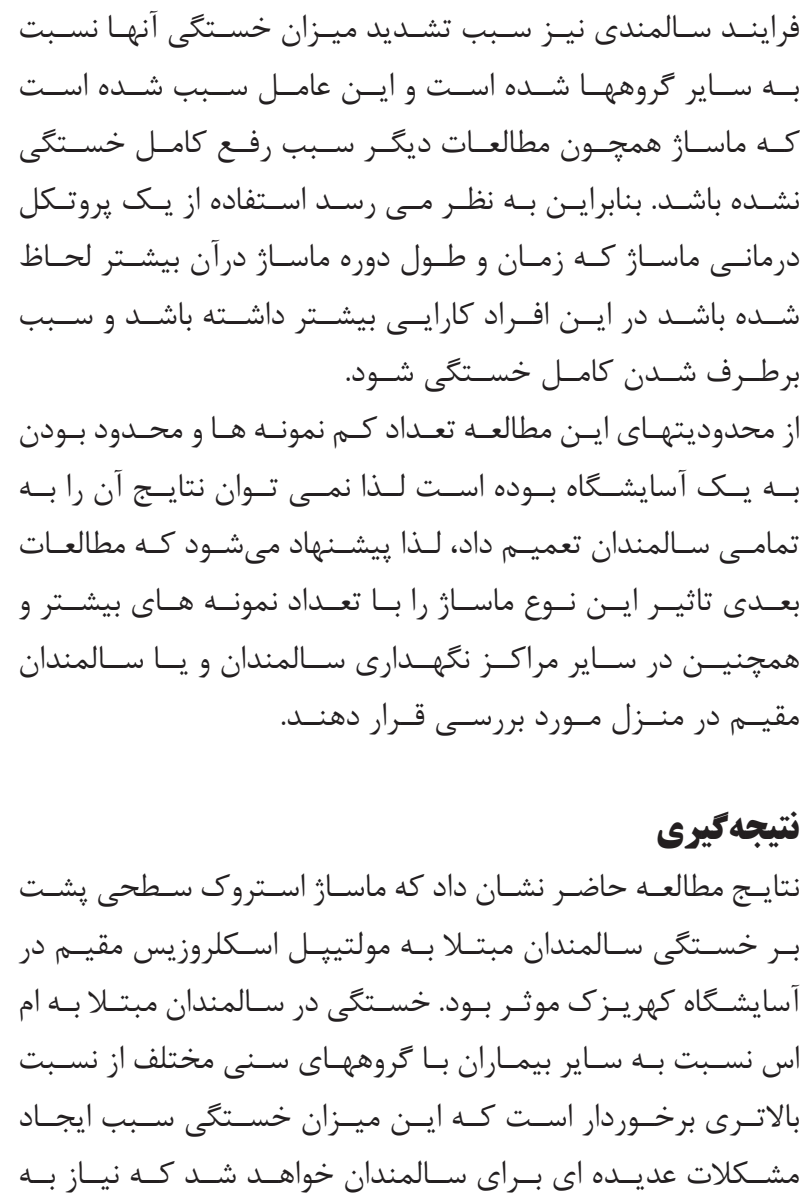

12. Braley TJ, Chervin RD. Fatigue in multiple sclerosis: mechanisms, evaluation, and treatment. Sleep. 2010;33(8):1061-7. PMID: 20815187

13. Ghaffari S, Ahmadi F, Nabavi SM, Kazem-Nezhad A. [Effects of applying hydrotherapy on quality of life in women with multiple sclerosis].J Rehabil. 2008;9(3):43-50.

14. Esmonde L, Long AF. Complementary therapy use by persons with multiple sclerosis: benefits and research priorities. Complement Ther Clin Pract. 2008;14(3):176-84. DOI: 10.1016/j.ctcp.2008.03.001 PMID: 18640629

15. Maa SH, Tsou TS, Wang KY, Wang CH, Lin HC, Huang YH. Self-administered acupressure reduces the symptoms that limit daily activities in bronchiectasis patients: pilot study findings. J Clin Nurs. 2007; 16(4):794-804. DOI: 10.1111/j.1365-2702.2005.01515.x PMID: 17402962

16. Buttagat V, Eungpinichpong W, Chatchawan U, Kharmwan S. The immediate effects of traditional Thai massage on heart rate variability and stress-related parameters in patients with back pain associated with myofascial trigger points. J Bodyw Mov Ther. 2011;15(1):15-23. DOI: 10.1016/j.jbmt.2009.06.005 PMID: 21147414

17. Harris M, Richards KC. The physiological and psychological effects of slow-stroke back massage and hand massage on relaxation in older people. J Clin Nurs. 2010;19(7-8):917-26. DOI: 10.1111/j.13652702.2009.03165.x PMID: 20492036

18. Field T, Diego M, Hernandez-Reif M. Massage therapy research. Developmen Rev. 2007;27(1):75-89.

19. Atashi V, Samiei F, Khatoni M, Alimoradi Z, Mojtabaipanah H. The effect of SSBM massage on anxiety and fatigue of patients white multiple sclerosis. J Appl Environ Biol Sci. 2014;4(8):217-23.

20. Shafiei Z, Nourian K, Babaee S, Nazari A. [Effectiveness of light pressure stroking massage on pain and fatigue of patients after coronary artery bypass graft surgery-A randomized clinical trial]. J Clin Nurs Midwif. 2013;2(3):28-38.

21. Karagozoglu S, Kahve E. Effects of back massage on chemotherapy-related fatigue and anxiety: supportive care and therapeutic touch in 
cancer nursing. Appl Nurs Res. 2013;26(4):210-7. DOI: 10.1016/j. apnr.2013.07.002 PMID: 24055114

22. Post-White J, Fitzgerald M, Savik K, Hooke MC, Hannahan AB, Sencer SF. Massage therapy for children with cancer. J Pediatr Oncol Nurs. 2009;26(1):16-28. DOI: $10.1177 / 1043454208323295$ PMID: 19074355

23. Ghafari S. Effect of relaxation on fatigue of patients with multiple sclerosis. J Shahrekord Uni Med Sci. 2008;10(1):61-8.

24. Shahvarughi Farahani A, Azimian M, Fallahpour M, Karimlou M. [Evaluation of reliability and validity of the Persian version of Fatigue
Severity Scale (FSS) among persons with multiple sclerosis]. J Rehabil. 2013;13(4):84-91.

25. Hagell P, Hoglund A, Reimer J, Eriksson B, Knutsson I, Widner H, et al. Measuring fatigue in Parkinson's disease: a psychometric study of two brief generic fatigue questionnaires. J Pain Symptom Manage. 2006;32(5):420-32. DOI: 10.1016/j.jpainsymman.2006.05.021 PMID: 17085268

26. Hasankhani H, Ghaderi F, Lakdizaji S, Nahamin M. The effect of the Slow-Stroke back massage on fatigue of dialyzed patients. Int Res J Appl Basic Sci. 2013;4(10):3004-08. 


\title{
The Effect of Slow Stroke Back Massage on Elderlies With Multiple Sclerosis in Kahrizak Charity Foundation in 2014
}

\author{
Mina Karimi ${ }^{1}$, Kian Nourozi Tabrizi ${ }^{2, *}$, Farahnaz Mohamadi ${ }^{3}$, \\ Ali Akbar Biglarian ${ }^{4}$
}

${ }^{1}$ MSc Student of Geriatric, Nursing Department, University of Social Welfare and Rehabilitation Sciences, Tehran, Iran

${ }^{2}$ Associate Professor, Nursing Department, University of Social Welfare and Rehabilitation Sciences, Tehran, Iran

${ }^{3}$ Assistant Professor, Nursing Department, University of Social Welfare and Rehabilitation Sciences, Tehran, Iran

${ }^{4}$ Assistant Professor, Statistics Department, University of Social Welfare and Rehabilitation Sciences, Tehran, Iran

* Corresponding author: Kian Nourozi Tabrizi, Associate Professor, Nursing Department, University of Social Welfare and Rehabilitation Sciences, Tehran, Iran.

Received: 09.01.2015 E-mail:dr.kian_nourozi@yahoo.com

Accepted: 03.19.2016

\section{Keywords:}

Multiple Sclerosis

Slow Stroke Back Massage

Fatigue

Elderly

(C) 2016 Iranian Nursing Scientific Association

\section{Abstract}

Introduction: Fatigue is the most common symptom of multiple sclerosis and the most common complaint in elderlies, which reduces the quality of life. This study aimed to evaluate the effect of slow stroke back massage on fatigue in elderly patients with multiple sclerosis living in Kahrizak Charity Foundation in 2014.

Methods: This was a quasi-experimental study, with one group before and after. The population consisted of 18 elderly male and female residents in Kahrizak Charity Foundation. Available sampling was performed based on the inclusion criteria, and for each individual, slow stroke back massage was applied for 20 minutes, in even days during a four-week period. Questionnaires were provided for demographic information and a questionnaire was used to assess fatigue. Fatigue was measured three times; before the intervention, at the end of the sixth session, and at the end of the twelfth session. Data Analysis was performed by SPSS version 16, and the statistical tests of Shapiro-Wilkes, repeated ANOVA and Friedman, Wilcoxon and Bonferroni, and U-Mann Whitney were used.

Results: Of the study population, $55.6 \%$ were female and $\% 44.4$ were male. The mean of age was 64.55 . The mean scores of fatigue in pre- and post-tests were 50.2 and 44.3 respectively, and it was 40.5 at the end of the sixth session. The results of this study showed that fatigue had a significant difference before and after the intervention and slow stroke back massage was effective for reducing fatigue $(\mathrm{P}<0.001)$.

Conclusions: Fatigue is highly prevalent in elderly patients with multiple sclerosis. The implementation of slow stroke back massage by caregivers and nurses, which is a tolerable and light intervention style for the elderlies, can be effective in reducing their fatigue. 